\title{
OVÁRIOS POLICÍSTICOS: CRITÉRIOS HEMODINÂMICOS
}

\author{
Márcio Augusto Pinto de Ávila ${ }^{1}$, Carlos Geraldo Viana Murta ${ }^{2}$
}

Resumo $\mathrm{O}$ advento da ultra-sonografia endovaginal de alta resolução abriu novas áreas de pesquisa nos ovários polićsticos. $O$ conhecimento da hemodinâmica ovariana é fundamental para o entendimento do comportamento fisiopatológico dos ovários policísticos. Os autores tecem considerações sobre a possibilidade da utilização do Doppler colorido na melhor definição dos ovários policísticos. Os dados sugerem que o aumento da vascularidade e a diminuição da resistência dos vasos do estroma ovariano, assim como o aumento do índice de pulsatilidade das artérias uterinas, possivelmente, são novos parâmetros sonográficos no diagnóstico da síndrome dos ovários policísticos. Esta é uma conclusão preliminar, e se fazem necessários estudos posteriores para confirmar esta proposição.

Unitermos: Ovário policístico. Ultra-sonografia. Doppler colorido. Fluxo sanguíneo.

\begin{abstract}
Polycystic ovaries: hemodynamic criteria.
The advent of high-resolution transvaginal ultrasound scanning introduced new research possibilities for patients with polycystic ovaries. The knowledge of the ovarian hemodynamics is a prerequisite for understanding the pathophysiological behaviour of polycystic ovaries. The authors consider the possibility of using color Doppler for a better definition of polycystic ovaries. The findings suggest that increased stromal blood vessels with low resistance and increased pulsatility index of the uterine arteries are to be new parameters to assist in the ultrasound diagnosis of polycystic ovary syndrome. This is a preliminary conclusion and further investigations are necessary to confirm this proposition.

Key words: Polycystic ovary. Ultrasonography. Color Doppler. Blood flow.
\end{abstract}

O acervo de combinações que constitui a síndrome dos ovários policísticos (SOP), tangíveis num extremo pela síndrome de Stein-Leventhal e, no oposto, pelos ovários policísticos (OP) livres de sinais exteriores, concorre para o contraste entre os resultados provenientes de diferentes métodos de estudo.

Doença plural e multifacetada, a SOP evidencia a heterogeneidade dos critérios clínicos, hormonais e anatômicos que compõem o seu espectro fenotípico. É uma doença com inter-relações em diversos campos da medicina, por vezes dissimulada, em outras exuberante, sem contudo restringir-se aos dados da imediata avaliação diagnóstica, mas com efeitos que se distanciam no longo prazo e, portanto, surpreendentes, quando não irredutíveis. Todavia, depreende-se da literatura a conceituação renovada a

1. Professor Doutor, Coordenador dos Cursos de Pós graduação da Universidade Federal do Rio de Janeiro (UFRJ), Rio de Janeiro, RJ.

2. Mestre pela UFRJ, Pós-graduando em Doutoramento na Universidade Federal de São Paulo/Escola Paulista de Medicina, Médico Ultra-sonografista da Universidade Federal do Espírito Santo.E-mail: cgvmurta@zaz.com.br

Endereço para correspondência: Prof. Dr. Márcio Augusto Pinto de Ávila. Rua Prudente de Moraes, 524/402, Ipanema. Rio de Janeiro, RJ, 22420-041. E-mail: marcioavila@openlink. com.br

Aceito para publicação em 23/10/2000. respeito do seu caráter evolutivo e a configuração anatômica que, identificada mediante o emprego da ultra-sonografia endovaginal, relegou o componente histopatológico, dado clássico da morfologia alterada. As modificações estruturais e não apenas aquelas atinentes à forma e ao tamanho são esmiuçadas, em especial o substrato intra-ovariano, folículos e estroma, núcleo dos distúrbios que mais oferecem pormenores. Além da definição proposta, a hipertrofia dos ovários policísticos abriga concepções distintas quanto ao número mínimo de folículos. Em contrapartida, atribui-se ao excesso de estroma, aspecto muito ou pouco incidente, relevância maior.

Não obstante as contradições acumuladas, no contexto dos parâmetros clínicos, concluiu-se que a hipertrofia dos ovários vincula-se à SOP em decorrência da preponderância do estroma, sítio em que o estudo da microcirculação passa a desempenhar importante linha de pesquisa. Observação primeira de Zaidi et al., em $1995^{(1)}$, descreve, na falta de irregularidades menstruais e endócrinas, o aumento do fluxo sanguíneo no estroma, com maior celeridade comparativamente aos ovários íntegros. O acréscimo da teia vascular, nos três dias iniciais do ciclo, por intermédio da exploração pelo Doppler colorido endovaginal e pulsátil, aliado à maior velocidade, favorecem a perspectiva de conceituação inédita dos OPs. Com o advento do "power" Doppler, por vez primeira, admitese a possibilidade do seu emprego nos OPs, o que proporciona melhor definição da vascularidade ovariana (Figura 1).

Battaglia et al. ${ }^{(2)}$ investigaram as variações dos fluxos intra-ovariano e uterino (artéria uterina). $\mathrm{O}$ aumento da relação LH/FSH, assim como das taxas de androstenediona, coincidiu com a baixa resistência ao fluxo no estroma (Figura 2) e, ao contrário, elevação do índice de pulsatilidade (IP) da artéria uterina (Figura 3). Aleem e Predanic ${ }^{(3)}$, em trabalho de 1996, comentam, a propósito de pacientes com SOP, a dissociação dos índices de resistência e pulsatilidade, menores no estroma e mais pronunciados nas artérias uterinas, comparados ao grupo com ciclos regulares e ovulação constante. A influência ponderal no discrime dos fluxos sanguíneos, ovariano e uterino, foi estudada por Battaglia et al. ${ }^{(4)}$. Vigente a SOP, o índice de massa corporal $\left(\right.$ IMC) $>25 \mathrm{~kg} / \mathrm{m}^{2}$ correspondeu ao aumento significativo do IP médio das 
artérias uterinas, prevalecente em relação ao valor detectado no grupo cuja magreza foi arbitrada pelo IMC $<23 \mathrm{~kg} /$ $\mathrm{m}^{2}$. No estroma ovariano, todavia, o comportamento hemodinâmico permaneceu independente dos fenótipos discrepantes (obesidade e magreza).

Battaglia et al. ${ }^{(5)}$, em 1998, comunicam os resultados preliminares, em pacientes com SOP, acerca da relação entre o conteúdo endócrino e o fluxo sanguíneo intra-ovariano e uterino com a distribuição dos folículos, generalizada e periférica. Concluem que as diferenças na morfologia ovariana não refletem padrões hormonais distintos e admitem a evolução morfológica proveniente da forma multicística normal, que termina na distribuição policística periférica, antecedida pelo estádio de dispersão folicular com predominância central. As diferenças na hemodinâmica intra-ovariana estiveram subordinadas à conformação dos folículos; menor o índice de resistência quando da localização periférica, em comparação ao grupo cístico, sem organização. Isento da destinação folicular, o IP médio elevado na artéria uterina mostrou correlação inversa com a taxa de estradiol no plasma e eqüidade com os níveis de androstenediona. A relação LH/FSH esteve em oposição ao menor índice pulsátil intra-ovariano e foi significativamente maior, a par da ecogenicidade mais exuberante, quando cingida pelo agrupamento cístico periférico.

Zaidi et al. ${ }^{(6)}$, em 1998, empreendem estudo prospectivo longitudinal a respeito das variações hemodinâmicas nos fluxos intra-ovariano e uterino, relacionados ao componente endócrino, definida a SOP e a ovulação sequiente ao uso de citrato de clomifeno. A velocidade sistólica máxima nos vasos do estroma e folículo permaneceu maior, à semelhança dos ovários e ciclos menstruais normais (Tan et al. ${ }^{(7)}$ ), assim como o IP sem diferenças significantes, tanto no estroma como na parede folicular, equiparável àquele observado em ciclos normais com ovulação espontânea. Considera-se improvável que a maior velocidade seja consecutiva aos efeitos do clomifeno, em virtude de a aceleração do fluxo no es-

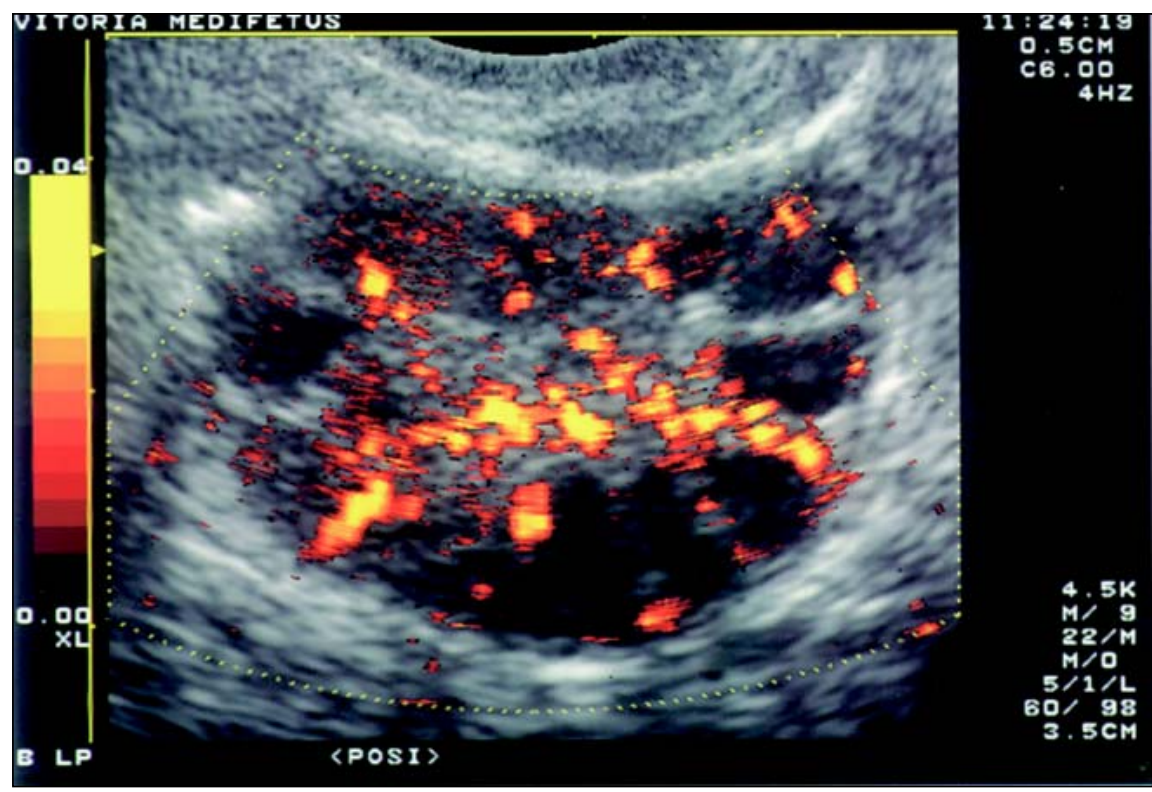

Figura 1. Observa-se riqueza de definição da vascularidade do ovário policístico à luz do Doppler de amplitude. Destaca-se o delineamento dos vasos interfoliculares.

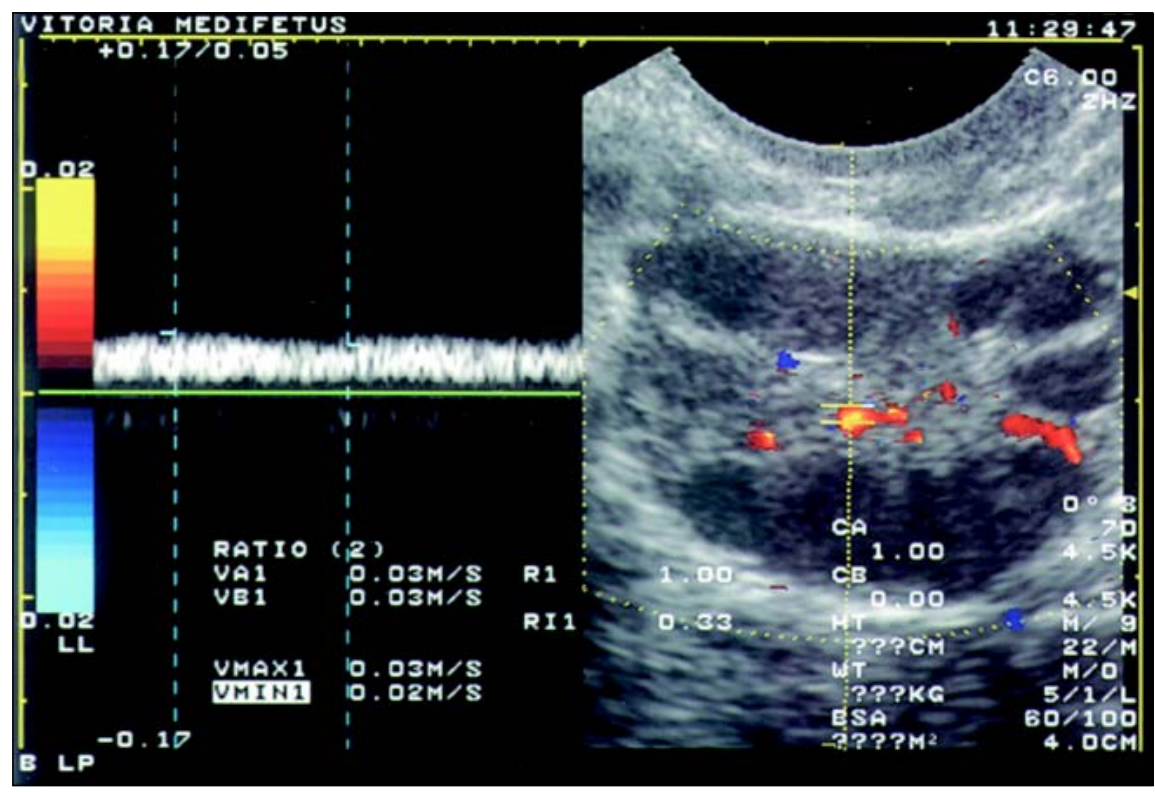

Figura 2. Onda de velocidade do fluxo sanguíneo mostrando vaso de baixa impedância (índice de resistência $=0,33$ ) no interior do estroma ovariano (ovário policístico) no quarto dia do ciclo.

troma e na parede folicular persistir e anteceder ao tratamento, o que sugere a interferência inata da SOP. O aumento da velocidade, contraposto à resistência inalterada, denota perfusão intra-ovariana crescente e, desse modo, maior o aporte de gonadotrofinas às células granulosas dos folículos em desenvolvimento. Parece viável, nesta circunstância, a resposta multifolicular e a incidência notável da síndrome de hiperestimulação ovariana (SHO). Evidentes os OPs, a angiogênese e a maior permeabilidade vascular condicionadas pelo fator de crescimento endotelial que, segundo Jacobs e Agrawal ${ }^{(8)}$, em concentrações expressivas após a administração de gonadotrofina coriônica, constitui-se no elo mais valioso na previsão da SHO.

Aglutina-se a experiência que assinala o aumento extraordinário do IP da artéria uterina correlacionado inversa- 


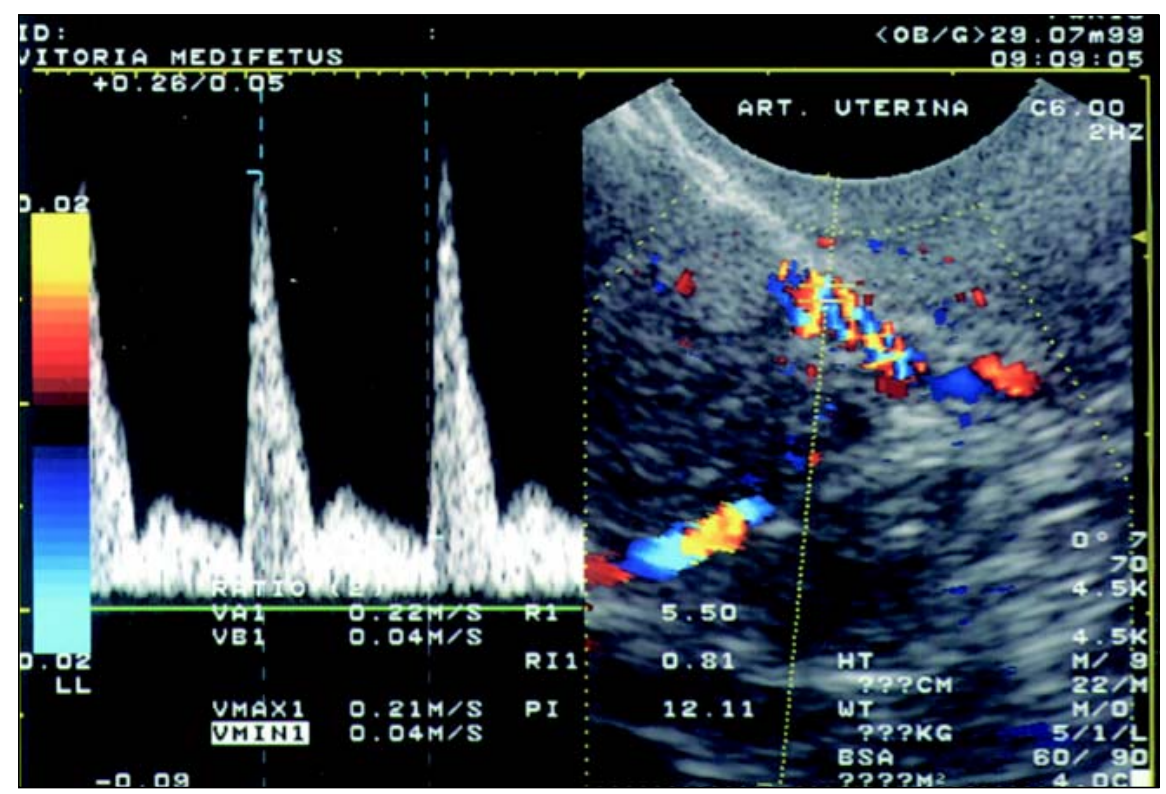

Figura 3. Onda de velocidade do fluxo da artéria uterina em paciente com ovário policístico no terceiro dia do ciclo menstrual, evidenciando alto índice de pulsatilidade $(I P=12)$.

mente com as concentrações de estradiol ao se divisar a SOP. Entretanto, Pinkas et al. ${ }^{(9)}$ referem, na artéria uterina, o fato inusitado e contrário aos dados precedentes de manter-se a pulsatilidade invariável em relação a ambos os tipos de ovários, policísticos ou normais.

Battaglia et al. ${ }^{(5)}$ comparam os efeitos da administração exclusiva de contraceptivo oral monofásico e sua associação com análogo do GnRH ("add-back therapy") em pacientes que apresentaram ovários policísticos além de hirsutismo, distúrbios menstruais e aumento dos androgênios. A provável maior gravidade desse grupo, cotejado ao de Pin- kas et al. ${ }^{(9)}$, reproduz experiências prévias que revelam o aumento da resistência ao fluxo na artéria uterina e o IP diminuído no estroma ovariano, fenômenos que se anteciparam à terapêutica e contrários à hemodinâmica natural dos ovários. Nota-se ainda, durante o tratamento, subtração do volume ovariano e declínio nas concentrações dos androgênios, circunstância em que comprovou-se a melhora do IP das artérias uterinas.

Em que pese o caráter experimental, o procedimento hemodinâmico constitui-se em mais um dado suscetível de incorporar-se à diversidade fenotípica da
SOP, em superposição ao critério morfológico. Por outro lado, os OPs assintomáticos, por intermédio da vascularidade acrescida e intensão da velocidade do fluxo sanguíneo no estroma, conferem interesse particular à compreensão da SHO.

\section{REFERÊNCIAS}

1. Zaidi J, Campbell S, Pittroff R, et al. Ovarian stromal blood flow in women with polycystic ovaries - a possible new marker for diagnosis? Hum Reprod 1995;10:1992-6.

2. Battaglia C, Artini PG, D'Ambrogio G, Genezzani $\mathrm{AD}$, Genezzani AR. The role of color Doppler imaging in the diagnosis of polycystic ovary syndrome. Am J Obstet Gynecol 1995; 172(1 Pt 1):10813.

3. Aleem FA, Predanic M. Transvaginal color Doppler determination of the ovarian and uterine blood flow characteristics in polycystic disease. Fertil Steril 1996;65:510-6.

4. Battaglia C, Artini PG, Genezzani AD, et al. Color Doppler analysis in lean and obese women with polycystic ovary syndrome. Ultrasound Obstet Gynecol 1996;7:342-6.

5. Battaglia C, Artini PG, Salvatori M, et al. Ultrasonographic patterns of polycystic ovaries: color Doppler and hormonal correlations. Ultrasound Obstet Gynecol 1998;11:332-6.

6. Zaidi J, Jacobs H, Campbell S, Tan SL. Blood flow changes in the ovarian and uterine arteries in women with polycystic ovary syndrome who respond to clomiphene citrate: correlation with serum hormone concentrations. Ultrasound Obstet Gynecol 1998;12:188-96.

7. Tan SL. Clinical applications of Doppler and threedimensional ultrasound in assisted reproductive technology. Ultrasound Obstet Gynecol 1999;13: $153-6$.

8. Jacobs HS, Agrawal R. Complications of ovarian stimulation. Baillière's Clin Obstet Gynaecol 1998; 12:565-79.

9. Pinkas H, Mashiach R, Rabinerson D, et al. Doppler parameters of uterine and ovarian stromal blood flow in women with polycystic ovary syndrome and normally ovulating women undergoing controlled ovarian stimulation. Ultrasound Obstet Gynecol 1998;12:197-200. 